\title{
Frequent Oscillation of a Class of Partial Difference Equations
}

\author{
C. J. Tian and B. G. Zhang
}

Abstract. This paper is concerned with the partial difference equation

$$
A_{m+1, n}+A_{m, n+1}-a_{m, n} A_{m, n}+p_{m, n} A_{m-k, n-1}=0
$$

where $k$ and $l$ are non-negative integers, $\left\{a_{m, n}\right\}$ and $\left\{p_{m, n}\right\}$ are real double sequences. Frequent oscillation criteria of this equation are obtained.

Keywords: Partial difference equations, frequent oscillation, frequent positivity

AMS subject classification: 39 A 10

\section{Introduction}

We consider the partial difference equation

$$
A_{m+1, n}+A_{m, n+1}-a_{m, n} A_{m, n}+p_{m, n} A_{m-k, n-l}=0
$$

where $\left\{a_{m, n}\right\}$ and $\left\{p_{m, n}\right\}$ are real double sequences, $m$ and $n$ are non-negative integers, $k$ and $l$ are positive integers. Equation (1.1) with $a_{m, n} \equiv 1$ has been studied in $[2,6,8$ - 11]. Partial difference equations arise from considerations of random walk problems, the study of molecular orbits [5], mathematical physics problems [8], and numerical difference approximation problems $[1,4]$. Difference equation $(1.1)$ can be obtained from the difference approximation of a class of delay partial differential equation of the form (see [4])

$$
\frac{\partial^{2} u}{\partial x^{2}}+\frac{\partial^{2} u}{\partial y^{2}}+g\left(x, y, u(x, y), u\left(x, y-\sigma_{1}\right), u\left(x-\tau_{1}, y\right), u\left(x-\tau_{2}, y-\sigma_{2}\right)\right)=0 .
$$

The oscillation of the above delay partial differential equation has been investigated by Tramov [7].

By a solution of equation (1.1) we mean a double sequence $\left\{A_{m, n}\right\}$ which is defined for $m \geq-k$ and $n \geq-l$ and satisfies (1.1) for $m \geq 0$ and $n \geq 0$.

C. J. Tian: South China Univ. Techn., Dept. of Elec. \& Comm. Eng., Guangzhou 510641, China

B. G. Zhang: Ocean Univ. of Qingdao, Dept. Appl. Math., Qingdao 266003, China

This work is supported by NNSF of China

ISSN 0232-2064 / \$ 2.50 C Heldermann Verlag Berlin 
The usual concept of oscillation of a sequence $\left\{A_{m, n}\right\}$ is the following: A solution $\left\{A_{i, j}\right\}$ of equation (1.1) is said to be eventually positive if $A_{i, j}>0$ for all large $i$ and $j$, and it is said to be oscillatory if it is neither eventually positive nor eventually negative.

However, the above definitions does not catch all the fine details of an oscillatory sequence. A strengthened oscillation which is called frequent oscillation has been posed by Tian et al. in [6]. In the present paper we will discuss frequent oscillation of solutions of equation (1.1).

\section{Preparatory lemmas}

For the sake of completeness, we list the following definitions which have been given in $[6]$.

The set $\{1,2, \cdots\}$ will be denoted by $\mathbb{N}$ and the set of integers by $\mathbb{Z}$. An element of $\mathbb{Z}^{2}=\mathbb{Z} \times \mathbb{Z}$ is called a lattice point. Let $\Omega$ be a set of lattice points, i.e. $\Omega \subset \mathbb{Z}^{2}$. The size of such set $\Omega$ will be denoted by $|\Omega|$. Given integers $m$ and $n$, the translation operators $X^{m}$ and $Y^{n}$ are defined by

$$
X^{m} \Omega=\left\{(i+m, j) \in \mathbb{Z}^{2} \mid(i, j) \in \Omega\right\} \quad \text { and } \quad Y^{n} \Omega=\left\{(i, j+n) \in \mathbb{Z}^{2} \mid(i, j) \in \Omega\right\}
$$

respectively. Let $\alpha, \beta$ and $\gamma, \tau$ be integers such that $\alpha \leq \beta$ and $\gamma \leq \tau$. The union $\sum_{i=\alpha}^{\beta} \sum_{j=\gamma}^{r} X^{i} Y^{j} \Omega$ is called a derived set of $\Omega$. Thus (see [6])

$$
\begin{gathered}
(i, j) \in \mathbb{Z}^{2} \backslash \sum_{i=\alpha}^{\beta} \sum_{j=\gamma}^{\tau} X^{i} Y^{j} \Omega \\
(i-k, j-l) \in \mathbb{Z}^{2} \backslash \Omega \text { for } \alpha \leq k \leq \beta \text { and } \gamma \leq l \leq \tau .
\end{gathered}
$$

Definition 2.1. Let $\Omega$ be a set of lattice points and

$$
\Omega^{(m, n)}=\{(i, j) \in \Omega \mid i \leq m \text { and } j \leq n\}
$$

If

$$
\limsup _{m, n \rightarrow \infty} \frac{\left|\Omega^{(m, n)}\right|}{m n}
$$

exists, then this limit denoted by $\mu^{*}(\Omega)$ will be called the upper frequent measure of $\Omega$. Similarly, if

$$
\liminf _{m, n \rightarrow \infty} \frac{\left|\Omega^{(m, n)}\right|}{m n}
$$

exists, then this limit denoted by $\mu_{*}(\Omega)$ will be called the lower frequent measure of $\Omega$. If $\mu^{*}=\mu_{*}$, then the common limit denoted by $\mu(\Omega)$ will be called the frequent measure of $\Omega$.

Definition 2.2. Let $X=\left\{x_{i, j}\right\}$ be a real double sequence. If $\mu^{*}(X \leq 0)=0$, then the sequence $X$ is said to be frequently positive, and if $\mu^{*}(X \geq 0)=0$, then $X$ is said to 
be frequently negative. The sequence $X$ is said to be frequently oscillatory if it is neither frequently positive nor frequently negative.

Obviously, if a double sequence is eventually positive, then it is frequently positive, and if it is eventually negative, then it is also frequently negative. Thus, if the sequence is frequently oscillatory, then it is oscillatory.

Definition 2.3. Let $X$ be a real double sequence. If $\mu^{*}(X \leq 0) \leq w$, then $X$ is said to be frequently positive of upper degree $w$, and if $\mu^{*}(X \geq 0) \leq w$, then $X$ is said to be frequently negative of upper degree $w$. The sequence $X$ is said to be frequently oscillatory of upper degree $w$ if it is neither frequently positive nor frequently negative of the same upper degree $w$. The concept of frequently positivity of lower degree etc. is similarly defined by means of $\mu_{*}$.

In order to show the difference of frequent oscillation and usual oscillation, let us see the following two examples in one dimension [6].

Example 2.1. Consider the sequence

$$
X=\left\{x_{k}\right\}_{k=1}^{\infty}=\{1,1,1,-1,1,1,1,-1, \cdots\} .
$$

Since $\mu_{*}(X \leq 0)=\frac{1}{4}$ and $\mu_{*}(X \geq 0)=\frac{3}{4}$, thus $X$ is frequently positive of lower degree $\frac{1}{4}$, frequently negative of lower degree $\frac{3}{4}$, and frequently oscillatory of lower degree $\frac{1}{5}$.

Example 2.2. The sequence

$$
x_{k}=\left\{\begin{array}{ll}
-1 & \text { if } k=2^{n} \\
+1 & \text { otherwise }
\end{array} \quad(n \in \mathbb{N})\right.
$$

is oscillatory, and frequently positive.

To prove our main results we need several preparatory results. The first three lemmas to show basic properties of frequency measures are taken from [6].

Lemma 2.1. Let $\Omega, \Gamma \subset \mathbb{N}^{2}$. Then

$$
\mu^{*}(\Omega+\Gamma) \leq \mu^{*}(\Omega)+\mu^{*}(\Gamma)
$$

Furthermore, if $\Omega$ and $\Gamma$ are disjoint, then

$$
\mu_{*}(\Omega)+\mu_{*}(\Gamma) \leq \mu_{*}(\Omega+\Gamma) \leq \mu_{*}(\Omega)+\mu^{*}(\Gamma) \leq \mu^{*}(\Omega+\Gamma) \leq \mu^{*}(\Omega)+\mu^{*}(\Gamma) .
$$

From Lemma 2.1 we can get

$$
\mu_{*}(\Omega)+\mu^{*}\left(\mathbb{N}^{2} \backslash \Omega\right)=1
$$

for any subset $\Omega$ of $\mathbb{N}^{2}$. 
Lemma 2.2. Let $\Omega, \Gamma \subset \mathbb{N}^{2}$. If $\mu^{*}(\Omega)+\mu_{*}(\Gamma)>1$, then $\Omega \cap \Gamma$ is an infinite set.

Lemma 2.3. Let $\Omega \subset \mathbb{N}^{2}$ and let $\alpha, \beta$ and $\gamma, \tau$ be integers such that $\alpha \leq \beta$ and $\gamma \leq \tau$. Then

$$
\mu_{*}\left(\sum_{i=\alpha}^{\beta} \sum_{j=\gamma}^{\tau} X^{i} Y^{j} \Omega\right) \leq(\beta-\alpha+1)(\tau-\gamma+1) \mu_{*}(\Omega)
$$

and

$$
\mu^{*}\left(\sum_{i=\alpha}^{\beta} \sum_{j=\gamma}^{\tau} X^{i} Y^{j} \Omega\right) \leq(\beta-\alpha+1)(\tau-\gamma+1) \mu^{*}(\Omega) .
$$

Lemma 2.4. Assume that $\left\{A_{m, n}\right\}$ is a solution of equation (1.1) such that

$$
\begin{aligned}
& A_{m, n}>0 \quad \text { for }\left\{\begin{array}{l}
m \in\{\bar{m}-2 k, \ldots, \bar{m}+\tau\} \\
n \in\{\bar{n}-2 l, \ldots, \bar{n}+\tau\}
\end{array}\right. \\
& p_{m, n} \geq \bar{p}>0 \quad \text { and } 0<a_{m, n} \leq \bar{a} \quad \text { for }\left\{\begin{array}{l}
m \in\{\bar{m}-k, \ldots, \bar{m}+\tau\} \\
n \in\{\bar{n}-l, \ldots, \bar{n}+\tau\}
\end{array}\right.
\end{aligned}
$$

where $\bar{m} \geq k, \bar{n} \geq l$ and $\tau \geq 2$ are positive integers. Then

$$
\begin{aligned}
\bar{a}^{r+1} A_{\bar{m}, \bar{n}} \geq & \sum_{i=0}^{\tau+1} C_{\tau+1}^{i} A_{\bar{m}+\tau+1-i, \bar{n}+i} \\
& +(\tau+1) \bar{p} \sum_{j=0}^{r} C_{\tau}^{j} A_{\bar{m}+r-k-j, \bar{n}+j-l} \\
& +\bar{p}^{2} \sum_{i=1}^{r} i \bar{a}^{r-i} \sum_{j=0}^{i-1} C_{i-1}^{j} A_{\bar{m}+i-1-j-2 k, \bar{n}+j-2 l} .
\end{aligned}
$$

Proof. From (1.1), we have

$$
A_{\bar{m}+1, \bar{n}}+A_{\bar{m}, \bar{n}+1}+p_{\bar{m}, \bar{n}} A_{\bar{m}-k, \bar{n}-l}=a_{\bar{m}, \bar{n}} A_{\bar{m}, \bar{n}} \leq \bar{a} A_{\bar{m}, \bar{n}}
$$

and, for $m \in\{\bar{m}-k, \ldots, \bar{m}+\tau\}$ and $n \in\{\bar{n}-l, \ldots, \bar{n}+\tau\}$

$$
A_{m+1, n}+A_{m, n+1}+p_{m, n} A_{m-k, n-1} \leq a_{m, n} A_{m, n} \leq \bar{a} A_{m, n} .
$$

Therefore,

$$
\begin{aligned}
A_{\bar{m}+2, \bar{n}}+A_{\bar{m}+1, \bar{n}+1}+p_{\bar{m}+1, \bar{n}} A_{\bar{m}+1-k, \bar{n}-l}=a_{\bar{m}+1, \bar{n}} A_{\bar{m}+1, \bar{n}} \leq \bar{a} A_{\bar{m}+1, \bar{n}} \\
A_{\bar{m}+1, \bar{n}+1}+A_{\bar{m}, \bar{n}+2}+p_{\bar{m}, \bar{n}+1} A_{\bar{m}-k, \bar{n}+1-l}=a_{\bar{m}, \bar{n}+1} A_{\bar{m}, \bar{n}+1} \leq \bar{a} A_{\bar{m}, \bar{n}+1} \\
A_{\bar{m}+1-k, \bar{n}-l}+A_{\bar{m}-k, \bar{n}+1-l}+p_{\bar{m}-k, \bar{n}-l} A_{\bar{m}-2 k, \bar{n}-2 l} \leq \bar{a} A_{\bar{m}-k, \bar{n}-l}
\end{aligned}
$$


From the last three inequalities, we have

$$
\begin{aligned}
\bar{a}^{2} A_{\bar{m}, \bar{n}} \geq & \bar{a}\left(A_{\bar{m}+1, \bar{n}}+A_{\bar{m}, \bar{n}+1}+p_{\bar{m}, \bar{n}} A_{\bar{m}-k, \bar{n}-1}\right) \\
\geq & A_{\bar{m}+2, \bar{n}}+2 A_{\bar{m}+1, \bar{n}+1}+A_{\bar{m}, \bar{n}+2}+\left(p_{\bar{m}+1, \bar{n}}+p_{\bar{m}, \bar{n}}\right) A_{\bar{m}+1-k, \bar{n}-l} \\
& +\left(p_{\bar{m}, \bar{n}+1}+p_{\bar{m}, \bar{n}}\right) A_{\bar{m}-k, \bar{n}+1-l}+p_{\bar{m}, \bar{n}} p_{\bar{m}-k, \bar{n}-l} A_{\bar{m}-2 k, \bar{n}-2 l},
\end{aligned}
$$

i.e.

$$
\begin{aligned}
\bar{a}^{2} A_{\bar{m}, \bar{n}} \geq & \sum_{i=0}^{2} C_{2}^{i} A_{\bar{m}+2-i, \bar{n}+i} \\
& +2 \bar{p} \sum_{j=0}^{1} C_{1}^{j} A_{\bar{m}+1-k-j, \bar{n}+j-l} \\
& +\bar{p}^{2} \sum_{i=1}^{1} i \bar{a}^{1-i} \sum_{j=0}^{i-1} C_{i-1}^{j} A_{\bar{m}+i-1-j-2 k, \bar{n}+j-2 l}
\end{aligned}
$$

From (1.1) we obtain

$$
\begin{aligned}
& A_{\bar{m}+3, \bar{n}}+A_{\bar{m}+2, \bar{n}+1}+p_{\bar{m}+2, \bar{n}} A_{\bar{m}+2-k, \bar{n}-1} \leq \bar{a} A_{\bar{m}+2, \bar{n}} \\
& A_{\bar{m}+2, \bar{n}+1}+A_{\bar{m}+1, \bar{n}+2}+p_{\bar{m}+1, \bar{n}+1} A_{\bar{m}+1-k, \bar{n}+1-1} \leq \bar{a} A_{\bar{m}+1, \bar{n}+1} \\
& A_{\bar{m}+1, \bar{n}+2}+A_{\bar{m}, \bar{n}+3}+p_{\bar{m}, \bar{n}+2} A_{\bar{m}-k, \bar{n}+2-l} \leq \bar{a} A_{\bar{m}, \bar{n}+2} \\
& A_{\bar{m}+2-k, \bar{n}-l}+A_{\bar{m}+1-k, \bar{n}+1-l}+p_{\bar{m}+1-k, \bar{n}-l} A_{\bar{m}+1-2 k, \bar{n}-2 l} \leq \bar{a} A_{\bar{m}+1-k, \bar{n}-l} \\
& A_{\bar{m}+1-k, \bar{n}+1-l}+A_{\bar{m}-k, \bar{n}+2-l}+p_{\bar{m}-k, \bar{n}+1-l} A_{\bar{m}-2 k, \bar{n}+1-2 l} \leq \bar{a} A_{\bar{m}-k, \bar{n}+1-l} \text {. }
\end{aligned}
$$

Hence

$$
\begin{aligned}
\bar{a}^{3} A_{\bar{m}, \bar{n}} \geq & A_{\bar{m}+3, \bar{n}}+3 A_{\bar{m}+2, \bar{n}+1}+3 A_{\bar{m}+1, \bar{n}+2}+A_{\bar{m}, \bar{n}+3} \\
& +\left(p_{\bar{m}+2, \bar{n}}+p_{\bar{m}+1, \bar{n}}+p_{\bar{m}, \bar{n}}\right) A_{\bar{m}+2-k, \bar{n}-l} \\
& +\left(2 p_{\bar{m}+1, \bar{n}+1}+p_{\bar{m}+1, \bar{n}}+p_{\bar{m}, \bar{n}+1}+2 p_{\bar{m}, \bar{n}}\right) A_{\bar{m}+1-k, \bar{n}+1-l} \\
& +\left(p_{\bar{m}, \bar{n}+2}+p_{\bar{m}, \bar{n}+1}+p_{\bar{m}, \bar{n}}\right) A_{\bar{m}-k, \bar{n}+2-l} \\
& +\bar{a} \bar{p}^{2} A_{\bar{m}-2 k, \bar{n}-2 l}+2 \bar{p}^{2} A_{\bar{m}+1-2 k, \bar{n}-2 l}+2 \bar{p}^{2} A_{\bar{m}-2 k, \bar{n}+1-2 l}
\end{aligned}
$$

In view of the conditions of Lemma 2.4, we have

$$
\begin{aligned}
\bar{a}^{3} A_{\bar{m}, \bar{n}} \geq & \sum_{i=0}^{3} C_{3}^{i} A_{\bar{m}+3-i, \bar{n}+i} \\
& +3 \bar{p} \sum_{j=0}^{2} C_{2}^{j} A_{\bar{m}+2-k-j, \bar{n}+j-l} \\
& +\bar{p}^{2} \sum_{i=1}^{2} i \bar{a}^{2-i} \sum_{j=0}^{i-1} C_{i-1}^{j} A_{\bar{m}+i-1-j-2 k, \bar{n}+j-2 l}
\end{aligned}
$$


Assume that

$$
\begin{aligned}
\bar{a}^{\tau} A_{\bar{m}, \bar{n}} \geq & \sum_{i=0}^{\tau} C_{\tau}^{i} A_{\bar{m}+r-i, \bar{n}+i} \\
& +\tau \bar{p} \sum_{j=0}^{\tau-1} C_{r-1}^{j} A_{\bar{m}+r-1-k-j, \bar{n}+j-l} \\
& +\bar{p}^{2} \sum_{i=1}^{r-1} i \bar{a}^{\tau-i-1} \sum_{j=0}^{i-1} C_{i-1}^{j} A_{\bar{m}+i-1-j-2 k, \bar{n}+j-2 l}
\end{aligned}
$$

Then, for $0 \leq i \leq \tau$ and $0 \leq j \leq \tau-1$, we have

$$
\begin{aligned}
& A_{\bar{m}+\tau+1-i, \bar{n}+i}+A_{\bar{m}+\tau-i, \bar{n}+1+i}+\bar{p} A_{\bar{m}+\tau-k-i, \bar{n}+i-l} \leq \bar{a} A_{\bar{m}+\tau-i, \bar{n}+i} \\
& A_{\bar{m}+r-k-j, \bar{n}+j-l} \\
& +A_{\bar{m}+\tau-1-k-j, \bar{n}+1+j-1}+\bar{p} A_{\bar{m}+\tau-1-2 k-j, \bar{n}+j-2 l} \leq \bar{a} A_{\bar{m}+r-1-k-j, \bar{n}+j-l .}
\end{aligned}
$$

Thus

$$
\begin{aligned}
\bar{a}^{\tau+1} & A_{\bar{m}, \bar{n}} \\
\geq & \sum_{i=0}^{\tau} C_{\tau}^{i}\left(A_{\bar{m}+\tau+1-i, \bar{n}+i}+A_{\bar{m}+\tau-i, \bar{n}+1+i}+\bar{p} A_{\bar{m}+\tau-k-i, \bar{n}+i-l}\right) \\
& +\tau \bar{p} \sum_{j=0}^{\tau-1} C_{\tau-1}^{j}\left(A_{\bar{m}+\tau-k-j, \bar{n}+j-l}\right. \\
& \left.+A_{\bar{m}+\tau-1-k-j, \bar{n}+1+j-l}+\bar{p} A_{\bar{m}+\tau-1-2 k-j, \bar{n}+j-2 l}\right) \\
& +\bar{p}^{2} \sum_{i=1}^{r-1} i \bar{a}^{\tau-i} \sum_{j=0}^{i-1} C_{i-1}^{j} A_{\bar{m}+i-1-j-2 k, \bar{n}+j-2 l} \\
= & A_{\bar{m}+\tau+1, \bar{n}}^{\tau} \\
& +\sum_{i=1}^{i} C_{\tau}^{i} A_{\bar{m}+\tau+1-i, \bar{n}+i} \\
& +\sum_{i=0}^{\tau-1} C_{\tau}^{i} A_{\bar{m}+\tau-i, \bar{n}+1+i}+A_{\bar{m}, \bar{n}+\tau+1} \\
& +\bar{p} \sum_{i=0}^{r} C_{\tau}^{i} A_{\bar{m}+\tau-k-i, \bar{n}+i-l}+\tau \bar{p}\left(A_{\bar{m}+\tau-k, \bar{n}-l}\right. \\
& +\sum_{j=1}^{\tau-1} C_{\tau-1}^{j} A_{\bar{m}+\tau-k-j, \bar{n}+j-l} \\
& \left.+\sum_{j=0}^{r-2} C_{\tau-1}^{j} A_{\bar{m}+\tau-1-k-j, \bar{n}+1+j-l}+A_{\bar{m}-k, \bar{n}+\tau-l}\right)
\end{aligned}
$$




$$
\begin{aligned}
& +\tau \bar{p}^{2} \sum_{j=0}^{r-1} C_{r-1}^{j} A_{\bar{m}+\tau-1-2 k-j, \bar{n}+j-2 l} \\
& +\bar{p}^{2} \sum_{i=1}^{r-1} i \bar{a}^{\tau-i} \sum_{j=0}^{i-1} C_{i-1}^{j} A_{\bar{m}+i-1-j-2 k, \bar{n}+j-21} \\
& =A_{\bar{m}+r+1, \bar{n}} \\
& +\sum_{i=1}^{\tau}\left(C_{\tau}^{i}+C_{\tau}^{i-1}\right) A_{\bar{m}+r+1-i, \bar{n}+i}+A_{\bar{m}, \bar{n}+r+1} \\
& +\bar{p} \sum_{i=0}^{r} C_{\tau}^{i} A_{\bar{m}+\tau-k-i, \bar{n}+i-l}+\tau \bar{p}\left(A_{\bar{m}+r-k, \bar{n}-l}\right. \\
& \left.+\sum_{j=1}^{r-1}\left(C_{\tau-1}^{j}+C_{\tau-1}^{j-1}\right) A_{\bar{m}+\tau-k-j, \bar{n}+j-1}+A_{\bar{m}-k, \bar{n}+\tau-l}\right) \\
& +\bar{p}^{2} \sum_{i=1}^{\tau} i \bar{a}^{\tau-i} \sum_{j=0}^{i-1} C_{i-1}^{j} A_{\bar{m}+r-1-2 k-j, \bar{n}+j-21} \\
& =\sum_{i=0}^{r+1} C_{\tau+1}^{i} A_{\bar{m}+\tau-1-i, \bar{n}+i} \\
& +(\tau+1) \bar{p} \sum_{j=0}^{\tau} C_{\tau}^{i} A_{\bar{m}+\tau-k-j, \bar{n}+j-l} \\
& +\bar{p}^{2} \sum_{i=1}^{T} i \bar{a}^{r-i} \sum_{j=0}^{i-1} C_{i-1}^{j} A_{\bar{m}+\tau-1-2 k-j, \bar{n}+j-2 l} .
\end{aligned}
$$

The proof is complete

The following two lemmas can easily be derived from Lemma 2.4 and their proofs are thus omitted.

Lemma 2.5. Assume that $\left\{A_{m, n}\right\}$ is a solution of equation (1.1) such that

$$
\begin{array}{r}
A_{m, n}>0 \quad \text { for }\left\{\begin{array}{l}
m \in\{\bar{m}-3 k, \ldots, \bar{m}+l\} \\
n \in\{\bar{n}-3 l, \ldots, \bar{n}+k\}
\end{array}\right. \\
p_{m, n} \geq 0 \text { and } 0<a_{m, n} \leq \bar{a} \quad \text { for }\left\{\begin{array}{l}
m \in\{\bar{m}-2 k, \ldots, \bar{m}+l-1\} \\
n \in\{\bar{n}-2 l, \ldots, \bar{n}+k-1\}
\end{array}\right.
\end{array}
$$

where $\bar{m} \geq 2 k$ and $\bar{n} \geq 2 l$ are positive integers. Then

$$
C_{k+l}^{l} A_{\bar{m}, \bar{n}} \leq \bar{a}^{k+l} A_{\bar{m}-k, \bar{n}-l} .
$$


Lemma 2.6. Assume that $\left\{A_{m, n}\right\}$ is a solution of equation (1.1) such that

$$
A_{m, n}>0 \quad \text { for }\left\{\begin{array}{l}
m \in\{\bar{m}-2 k-l, \ldots, \bar{m}+2 k+l+1\} \\
n \in\{\bar{n}-2 l-k, \ldots, \bar{n}+k+2 l+1\}
\end{array}\right.
$$

$p_{m, n} \geq \bar{p} \quad$ and $\quad 0<a_{m, n} \leq \bar{a} \quad$ for $\left\{\begin{array}{l}m \in\{\bar{m}-k-l, \ldots, \bar{m}+2 k+l\} \\ n \in\{\bar{n}-k-l, \ldots, \bar{n}+k+2 l\}\end{array}\right.$

where $\bar{m}, \bar{n} \geq k+l$ are positive integers. Then

$$
\bar{a}^{k+l+1} A_{\bar{m}-h, \bar{n}+h} \geq(k+l+1) \bar{p} C_{k+l}^{l-h} A_{\bar{m}, \bar{n}} .
$$

for $-k \leq h \leq l$.

Lemma 2.7. Assume that the conditions of Lemma 2.4 hold and $\bar{p}=0$. Then

$$
\bar{a}^{r+1} A_{\bar{m}, \bar{n}} \geq \sum_{i=0}^{\tau+1} C_{\tau+1}^{i} A_{\bar{m}+\tau+1-i, \bar{n}+i}+\sum_{j=0}^{\tau}\left(\sum_{s=0}^{r-j} \sum_{t=0}^{j} p_{\bar{m}+s, \bar{n}+t}\right) A_{\bar{m}+\tau-k-j, \bar{n}+j-l} .
$$
that

Proof. As in the proof of Lemma 2.1, we know that inequality (2.5) holds. Assume

$$
\bar{a}^{r} A_{\bar{m}, \bar{n}} \geq \sum_{i=0}^{r} C_{\tau}^{i} A_{\bar{m}+\tau-i, \bar{n}+i}+\sum_{j=0}^{r-1}\left(\sum_{s=0}^{r-1-j} \sum_{t=0}^{j} p_{\bar{m}+s, \bar{n}+\imath}\right) A_{\bar{m}+r-k-j-1, \bar{n}+j-l}
$$

Then, for $0 \leq i \leq \tau$ and $0 \leq j \leq \tau-1$, we have

$$
\begin{aligned}
& A_{\bar{m}+r-k-j, \bar{n}+j-l}+A_{\bar{m}+r-k-1-j, \bar{n}+1+j-l} \leq \bar{a} A_{\bar{m}+r-1-k-j, \bar{n}+j-l} \\
& A_{\bar{m}+r+1-i, \bar{n}+i}+A_{\bar{m}+r-i, \bar{n}+1+i}+p_{\bar{m}+r-i, \bar{n}+i} A_{\bar{m}+r-i-k, \bar{n}+i-l} \leq \bar{a} A_{\bar{m}+r-i, \bar{n}+i} .
\end{aligned}
$$

Thus

$$
\begin{aligned}
& \bar{a}^{r+1} A_{\bar{m}, \bar{n}} \\
& \geq \sum_{i=0}^{r} C_{r}^{i}\left(A_{\bar{m}+\tau+1-i, \bar{n}+i}+A_{\bar{m}+r-i, \bar{n}+1+i}+p_{\bar{m}+r-i, \bar{n}+i} A_{\bar{m}+r-i-k, \bar{n}+i-1}\right) \\
& +\sum_{j=0}^{r-1}\left(\sum_{s=0}^{\tau-1-j} \sum_{t=0}^{j} p_{\bar{m}+s, \bar{n}+t}\right)\left(A_{\bar{m}+\tau-k-j, \bar{n}+j-l}+A_{\bar{m}+\tau-k-1-j, \bar{n}+1+j-l}\right) \\
& =\sum_{i=0}^{r+1} C_{r+1}^{i} A_{\bar{m}+r+1-i, \bar{n}+i}+\sum_{i=0}^{r} C_{\tau}^{i} p_{\bar{m}+r-i, \bar{n}+i} A_{\bar{m}+r-i-k, \bar{n}+i-l} \\
& +\sum_{s=0}^{r-1} p_{\bar{m}+s, \bar{n}} A_{\bar{m}+r-k, \bar{n}-l}+\sum_{j=1}^{r-1}\left(\sum_{s=0}^{r-1-j} \sum_{\imath=0}^{j} p_{\bar{m}+s, \bar{n}+\imath}\right) A_{\bar{m}+r-k-j, \bar{n}+j-\ell} \\
& +\sum_{j=0}^{r-2}\left(\sum_{s=0}^{r-1-j} \sum_{t=0}^{j} p_{\bar{m}+s, \bar{n}+t}\right) A_{\bar{m}+r-k-1-j, \bar{n}+1+j-l}+\sum_{t=0}^{r-1} p_{\bar{m}, \bar{n}+t} A_{\bar{m}-k, \bar{n}+r-l}
\end{aligned}
$$




$$
\begin{aligned}
& \geq \sum_{i=0}^{r+1} C_{\tau+1}^{i} A_{\bar{m}+r+1-i, \bar{n}+i}+\sum_{i=1}^{r-1} p_{\bar{m}+\tau-i, \bar{n}+i} A_{\bar{m}+r-i-k, \bar{n}+i-l} \\
& +p_{\bar{m}+r, \bar{n}} A_{\bar{m}+\tau-k, \bar{n}-1}+p_{\bar{m}, \bar{n}+r} A_{\bar{m}-k, \bar{n}+r-1} \\
& +\sum_{s=0}^{r-1} p_{\bar{m}+s, \bar{n}} A_{\bar{m}+r-k, \bar{n}-l}+\sum_{t=0}^{r-1} p_{\bar{m}, \bar{n}+t} A_{\bar{m}-k, \bar{n}+r-l} \\
& +\sum_{j=1}^{r-1}\left(\sum_{s=0}^{r-1-j} \sum_{t=0}^{j} p_{\bar{m}+s, \bar{n}+t}+\sum_{s=0}^{r-j} \sum_{t=0}^{j-1} p_{\bar{m}+s, \bar{n}+t}\right) A_{\bar{m}+r-k-j, \bar{n}+j-t} \\
& \geq \sum_{i=0}^{r+1} C_{r+1}^{i} A_{\bar{m}+r+1-i, \bar{n}+i}+\sum_{s=0}^{r} p_{\bar{m}+s, \bar{n}} A_{\bar{m}+r-k, \bar{n}-1} \\
& +\sum_{t=0}^{\tau} p_{\bar{m}, \bar{n}+t} A_{\bar{m}-k, \bar{n}+\tau-1}+\sum_{j=1}^{r-1}\left(p_{\bar{m}+\tau-j, \bar{n}+j}\right. \\
& \left.+\sum_{s=0}^{r-1-j} \sum_{t=0}^{j} p_{\bar{m}+s, \bar{n}+t}+\sum_{s=0}^{r-j} \sum_{t=0}^{j-1} p_{\bar{m}+s, \bar{n}+t}\right) A_{\bar{m}+r-k-j, \bar{n}+j-t} \\
& \geq \sum_{i=0}^{r+1} C_{\tau+1}^{i} A_{\bar{m}+r+1-i, \bar{n}+i}+\sum_{j=0}^{r}\left(\sum_{s=0}^{r-j} \sum_{t=0}^{j} p_{\bar{m}+s, \bar{n}+\imath}\right) A_{\bar{m}+r-k-j, \bar{n}+j-l} .
\end{aligned}
$$

The proof is complete

Lemma 2.8. If $0<a_{m, n} \leq 1$ and $p_{m, n} \geq 0$ for all large $m$ and $n$, then every non-oscillatory solution of equation (1.1) tends to zero as $m, n \rightarrow \infty$.

Proof. Assume that $\left\{A_{m, n}\right\}$ is an eventually positive solution of equation (1.1). Then there exist positive integers $\bar{m} \geq-3 k$ and $\bar{n} \geq-3 l$ such that

$$
\begin{aligned}
& A_{m, n}>0 \quad \text { for }\left\{\begin{array}{l}
m \geq \bar{m}-3 k \\
n \geq \bar{n}-3 l
\end{array}\right. \\
& 0<a_{m, n} \leq 1 \quad \text { and } \quad p_{m, n} \geq 0 . \text { for }\left\{\begin{array}{l}
m \geq \bar{m}-2 k \\
n \geq \bar{n}-2 l .
\end{array}\right.
\end{aligned}
$$

By Lemma 2.4, for positive integers $\tau_{1}$ and $\tau_{2}$ we have

$$
A_{\bar{m}, \bar{n}} \geq C_{r_{1}+r_{2}}^{r_{2}} A_{\bar{m}+r_{1}, \bar{n}+r_{2}} \text {. }
$$

Thus

$$
A_{\bar{m}+r_{1}, \bar{n}+r_{2}} \leq \frac{A_{\bar{m}, \bar{n}}}{C_{r_{1}+r_{2}}^{r_{2}}} \rightarrow 0 \quad \text { as } \tau_{1}, \tau_{2} \rightarrow \infty .
$$

The proof is complete

Lemma 2.9. Assume that for some positive integers $\bar{m}$ and $\bar{n}$, equation (1.1) has a solution $\left\{A_{m, n}\right\}$ such that

$$
A_{m, n}>0 \quad \text { for }\left\{\begin{array}{l}
\bar{m}-3 k \leq m \leq \bar{m}+l+k+1 \\
\bar{n}-3 l \leq n \leq \bar{n}+k+l+1
\end{array}\right.
$$


If

$$
\begin{aligned}
p_{m, n} \geq 0 \text { and } 0<a_{m, n} \leq \bar{a} & \text { for }\left\{\begin{array}{l}
\bar{m}-2 k \leq m \leq \bar{m}+l+k \\
\bar{n}-2 l \leq n \leq \bar{n}+k+l
\end{array}\right. \\
\sum_{i=m-k}^{m} \sum_{j=n-l}^{n} p_{i, j} \geq B>0 & \text { for }\left\{\begin{array}{l}
\bar{m} \leq m \leq \bar{m}+k \\
\bar{n} \leq n \leq \bar{n}+l,
\end{array}\right.
\end{aligned}
$$

then

$$
\frac{A_{\bar{m}-k, \bar{n}-l}}{A_{\bar{m}, \bar{n}}}<\left(\frac{2}{B}\right)^{4} \bar{a}^{3 k+3 l+4} .
$$

Proof. It is easy to see that there exists $m^{*}$ such that $\bar{m} \in\left\{m^{*}-k, \ldots, m^{*}\right\}$ and

$$
\sum_{i=m^{*}-k}^{\bar{m}} \sum_{j=\bar{n}-l}^{\bar{n}} p_{i, j} \geq \frac{B}{2} \quad \text { and } \quad \sum_{i=\bar{m}}^{m^{*}} \sum_{j=\bar{n}-1}^{\hat{n}} p_{i, j} \geq \frac{B}{2} .
$$

By Lemma 2.7, we have

$$
\bar{a}^{m^{*}-\bar{m}+l+1} A_{\bar{m}, \bar{n}-l} \geq\left(\sum_{s=0}^{m^{*}-\bar{m}} \sum_{\imath=0}^{l} p_{\bar{m}+s, \bar{n}+\imath-l}\right) A_{m^{*}-k, \bar{n}-l} \geq \frac{B}{2} A_{m^{*}-k, n-l}
$$

and

$$
\bar{a}^{\bar{m}-m^{*}+k+1+1} A_{m^{*}-k, \bar{n}-l} \geq\left(\sum_{s=0}^{\bar{m}-m^{\cdot}+k} \sum_{i=0}^{l} p_{m^{*}-k+s, \bar{n}-1+t}\right) A_{\bar{m}-k, \bar{n}-l} \geq \frac{B}{2} A_{\bar{m}-k, \bar{n}-l} .
$$

Thus

$$
A_{\bar{m}, \bar{n}-l} \geq\left(\frac{B}{2}\right)^{2} \bar{a}^{-k-2 l-2} A_{\bar{m}-k, \bar{n}-l} .
$$

Similarly, there exists $n^{*}$ such that $\bar{n} \in\left\{n^{*}-l, \ldots, n^{*}\right\}$ and

$$
\sum_{i=\bar{m}}^{\bar{m}+k} \sum_{j=n^{*}-1}^{\bar{n}} p_{i, j} \geq \frac{B}{2} \quad \text { and } \quad \sum_{i=\bar{m}}^{\bar{m}+k} \sum_{j=\bar{n}}^{n^{*}} p_{i, j} \geq \frac{B}{2} \text {. }
$$

By Lemma 2.7, we have

$$
\bar{a}^{n^{*}-\dot{n}+k+1} A_{\bar{m}, \bar{n}} \geq\left(\sum_{s=0}^{k} \sum_{t=0}^{n^{*}-\bar{n}} p_{\bar{m}+s, \bar{n}+t}\right) A_{\bar{m}, n^{*}-l} \geq \frac{B}{2} A_{\bar{m}, n^{*}-l}
$$

and

$$
\bar{a}^{\bar{n}-n^{*}+k+l+1} A_{\bar{m}, n^{*}-1} \geq\left(\sum_{s=0}^{k} \sum_{t=0}^{\bar{n}-n^{*}+l} p_{\bar{m}+s, n^{*}-l+t}\right) A_{\bar{m}, \bar{n}-l} \geq \frac{B}{2} A_{\bar{m}, \bar{n}-l} .
$$

Hence

Therefore

$$
A_{\bar{m}, \bar{n}} \geq\left(\frac{B}{2}\right)^{2} \bar{a}^{-1-2 k-2} A_{\bar{m}, \bar{n}-l}
$$

The proof is complete

$$
\frac{A_{\bar{m}-k, \bar{n}-l}}{A_{\bar{m}, \bar{n}}}=\frac{A_{\bar{m}-k, \bar{n}-l}}{A_{\bar{m}, \bar{n}-l}} \frac{A_{\bar{m}, \bar{n}-l}}{A_{\bar{m}, \bar{n}}}<\left(\frac{2}{B}\right)^{4} \bar{a}^{3 k+3 l+4} .
$$


The following fact is also an easy consequence of Lemma 2.4 and its proof is thus omitted.

Lemma 2.10. Assume that for positive integers $\bar{m}, \bar{n}$ and $\tau$ equation (1.1) has a solution $\left\{A_{m, n}\right\}$ such that

$$
\begin{array}{r}
A_{m, n}>0 \quad \text { for }\left\{\begin{array}{l}
m \in\{\bar{m}-k, \ldots, \bar{m}+\tau\} \\
n \in\{\bar{n}-l, \ldots, \bar{n}+\tau\}
\end{array}\right. \\
p_{m, n} \geq 0 \text { and } 0<a_{m, n} \leq \bar{a} \quad \text { for }\left\{\begin{array}{l}
m \in\{\bar{m}, \ldots, \bar{m}+\tau\} \\
n \in\{\bar{n}, \ldots, \bar{n}+\tau\} .
\end{array}\right.
\end{array}
$$

Then

$$
\bar{a}^{r} A_{\bar{m}, \bar{n}}>A_{\bar{m}+r, \bar{n}} \quad \text { and } \quad \bar{a}^{r} A_{\bar{m}, \bar{n}}>A_{\bar{m}, \bar{n}+r}
$$

\section{Main results}

Let $a=\left\{a_{m, n}\right\}$ and $p=\left\{p_{m, n}\right\}$ be real double sequences.

Theorem 3.1. Assume that $\bar{p}$ and $\bar{a}$ are two positive real numbers and $w$ a nonnegative number. Let $\mu^{*}(a>\bar{a}$ or $a \leq 0)=\bar{A}$ and $\mu^{*}(p<\bar{p})=\bar{B}$. Suppose further that

$$
\bar{p} \frac{(k+l+1) !}{k ! l ! \bar{a}^{k+l+1}} \geq 1
$$

and

$$
(2 k+l+1)(2 l+k+1)(\bar{A}+\bar{B})+(3 k+l+2)(3 l+k+2) w<1 .
$$

Then every solution of equation (1.1) is frequently oscillatory of lower degree $w$.

Proof. Suppose to the contrary there is a frequently positive solution $A=\left\{A_{m, n}\right\}$ of equation (1.1) with lower degree $w$ such that $\mu_{*}(A \leq 0) \leq w$. In view of Lemmas 2.1 - (2.4) we have

$$
\begin{aligned}
\mu *\left(\mathbb{N}^{2}\right. & \left.\sum_{i=-k-l} \sum_{j=-k-1}^{l} X^{i} Y^{j}(a>\bar{a} \text { or } p<\bar{p})\right) \\
& +\mu^{*}\left(\mathbb{N}^{2} \backslash \sum_{i=-k-l-1}^{2 k} \sum_{j=-k-l-1}^{2 l} X^{i} Y^{j}(A \leq 0)\right) \\
= & 2-\mu^{*}\left(\sum_{i=-k-l}^{k} \sum_{j=-k-l}^{l} X^{i} Y^{j}(a>\bar{a} \text { or } p<\bar{p})\right) \\
& -\mu_{*}\left(\sum_{i=-k-l-1}^{2 k} \sum_{j=-k-l-1}^{2 l} X^{i} Y^{j}(A \leq 0)\right) \\
\geq & 2-(2 k+l+1)(2 l+k+1) \mu^{*}(a>\bar{a} \text { or } p<\bar{p}) \\
& -(3 k+l+2)(3 l+k+2) \mu_{*}(A \leq 0)
\end{aligned}
$$




$$
\begin{aligned}
& \geq 2-(2 k+l+1)(2 l+k+1)(\bar{A}+\bar{B})-(3 k+l+2)(3 l+k+2) w \\
& >1 .
\end{aligned}
$$

Hence, by Lemma 2.2,

$$
\begin{aligned}
\left(\mathbb{N}^{2} \backslash\right. & \left.\sum_{i=-k-l}^{k} \sum_{j=-k-1}^{l} X^{i} Y^{j}(a>\bar{a} \text { or } p<\bar{p})\right) \\
& \bigcap\left(\mathbb{N}^{2} \backslash \sum_{i=-k-l-1}^{2 k} \sum_{j=-k-l-1}^{2 l} X^{i} Y^{j}(A \leq 0)\right)
\end{aligned}
$$

is an infinite subset of $\mathbb{N}^{2}$, which together with (2.1), imply that there is a lattice point $(\bar{m}, \bar{n})$ such that

$$
\begin{array}{r}
A_{m, n}>0 \quad \text { for }\left\{\begin{array}{l}
\bar{m}-2 k \leq m \leq \bar{m}+k+l+1 \\
\bar{n}-2 l \leq n \leq \bar{n}+k+l+1
\end{array}\right. \\
0<a_{m, n} \leq \bar{a} \text { and } p_{m, n} \geq \bar{p} \text { for }\left\{\begin{array}{l}
\bar{m}-k \leq m \leq \bar{m}+k+l \\
\bar{n}-l \leq n \leq \bar{n}+k+l .
\end{array}\right.
\end{array}
$$

By Lemma 2.4, we obtain $\bar{a}^{k+l+1} A_{\bar{m}, \bar{n}}>(k+l+1) \bar{p} C_{k+1}^{l} A_{\bar{m}, \bar{n}}$. Hence

$$
\bar{p} \frac{(k+l+1) !}{k ! ! ! \bar{a}^{k+l+1}}<1
$$

which is a contradiction. The proof is complete

Lemma 3.1. Let $k>0, l>0, \Psi>0, \bar{a}>0$ and

$$
\Gamma=\bar{a}^{k+l+1} \lambda^{\lambda} 2^{-\lambda}(1+\lambda)^{-1-\lambda} \quad \text { where } \lambda=2 k l(k+l)^{-1} .
$$

Assume that for some positive integers $\bar{m}, \bar{n}$ and $\tau$ equation (1.1) has a solution $\left\{A_{m, n}\right\}$ such that

$$
\begin{aligned}
A_{i, j}>0 & \text { for }\left\{\begin{array}{l}
\bar{m}-(\tau+3) k \leq i \leq \bar{m}+l \\
\bar{n}-(\tau+3) l \leq j \leq \bar{n}+k
\end{array}\right. \\
0<a_{i, j} \leq \bar{a} \text { and } p_{i, j} \geq 0 & \text { for }\left\{\begin{array}{l}
\bar{m}-(\tau+2) k \leq i \leq \bar{m}+l \\
\bar{n}-(\tau+2) l \leq j \leq \bar{n}+k
\end{array}\right. \\
\frac{1}{k l} \sum_{i=m-k}^{m-1} \sum_{j=n-l}^{n-1} p_{i, j} \geq \Psi & \text { for }\left\{\begin{array}{l}
\bar{m}-(\tau-1) k \leq m \leq \bar{m} \\
\bar{n}-(\tau-1) l \leq n \leq \bar{n} .
\end{array}\right.
\end{aligned}
$$

Then

$$
\frac{A_{\bar{m}, \bar{n}}}{A_{\bar{m}-k, \bar{n}-l}} \leq \frac{\bar{a}^{k+l}}{C_{k+l}^{l}}\left(\frac{\Gamma}{\Psi}\right)^{\top} .
$$


Proof. From (1.1) and Lemma 2.5, we have

$$
\begin{aligned}
A_{m+1, n}+A_{m, n+1}-\bar{a} A_{m, n} & \leq A_{m+1, n}+A_{m, n+1}-a_{m, n} A_{m, n} \\
& =-p_{m, n} A_{m-k, n-l} \\
& \leq-p_{m, n} \frac{C_{k+l}^{l}}{\bar{a}^{k+1}} A_{m, n}
\end{aligned}
$$

for $\bar{m}-\tau k \leq m \leq \bar{m}$ and $\bar{n}-\tau l \leq n \leq \bar{n}$. Thus

$$
p_{m, n} \leq \frac{\bar{a}^{k+l}}{C_{k+1}^{l}}\left(\bar{a}-\frac{A_{m+1, n}+A_{m, n+1}}{A_{m, n}}\right) \leq \frac{\bar{a}^{k+l}}{C_{k+l}^{l}}\left(\bar{a}-\frac{2\left(A_{m+1, n} A_{m, n+1}\right)^{\frac{1}{2}}}{A_{m, n}}\right) .
$$

Hence, for $\bar{m}-(\tau-1) k \leq m \leq \bar{m}$ and $\bar{n}-(\tau-1) l \leq n \leq \bar{n}$ we have

$$
\begin{aligned}
\Psi & \leq \frac{1}{k l} \sum_{i=m-k}^{m-1} \sum_{j=n-l}^{n-1} p_{i, j} \\
& \leq \frac{\bar{a}^{k+l}}{C_{k+l}^{l}} \frac{1}{k l} \sum_{i=m-k}^{m-1} \sum_{j=n-l}^{n-1}\left(\bar{a}-\frac{2\left(A_{i+1, j} A_{i, j+1}\right)^{\frac{1}{2}}}{A_{i, j}}\right) \\
& =\frac{\bar{a}^{k+l}}{C_{k+l}^{l}}\left(\bar{a}-\frac{2}{k l} \sum_{i=m-k}^{m-1} \sum_{j=n-l}^{n-1} \frac{\left(A_{i+1, j} A_{i, j+1}\right)^{\frac{1}{2}}}{A_{i, j}}\right) .
\end{aligned}
$$

Note that by the inequality between the arithmetic and geometric means and Lemma 2.10 we have

$$
\begin{aligned}
& \sum_{i=m-k}^{m-1} \sum_{j=n-l}^{n-1} \frac{\left(A_{i+1, j} A_{i, j+1}\right)^{\frac{1}{2}}}{A_{i, j}} \\
& \geq \sum_{i=m-k}^{m-1} l\left(\prod_{j=n-l}^{n-1} \frac{\left(A_{i+1, j} A_{i, j+1}\right)^{\frac{1}{2}}}{A_{i, j}}\right)^{\frac{1}{i}} \\
& =l \sum_{i=m-k}^{m-1}\left(\frac{A_{i, n}}{A_{i, n-1}} \prod_{j=n-1}^{n-1} \frac{A_{i+1, j}}{A_{i, j}}\right)^{\frac{1}{21}} \\
& \geq k l \prod_{i=m-k}^{m-1}\left(\frac{A_{i, n}}{A_{i, n-1}}\right)^{\frac{1}{2 k}} \prod_{i=m-k}^{m-1} \prod_{j=n-1}^{n-1} \cdot\left(\frac{A_{i+1, j}}{A_{i, j}}\right)^{\frac{1}{2 k}} \\
& =k l \prod_{i=m-k}^{m-1}\left(\frac{A_{i, n}}{A_{i, n-l}}\right)^{\frac{1}{2 k l}} \prod_{j=n-1}^{n-1}\left(\frac{A_{m, j}}{A_{m-k, j}}\right)^{\frac{1}{2 k T}} \\
& \geq k l \prod_{i=m-k}^{m-1}\left(\frac{A_{m, n}}{\bar{a}^{m-i}} \frac{1}{\bar{a}^{i-m+k} A_{m-k, n-l}}\right)^{\frac{1}{2 k l}} \prod_{j=n-l}^{n-1}\left(\frac{1}{\bar{a}^{l}} \frac{A_{m, n}}{A_{m-k, n-l}}\right)^{\frac{1}{2 k l}}
\end{aligned}
$$




$$
\begin{aligned}
& =k l \bar{a}^{-\frac{k^{2}+l^{2}}{2 k l}}\left(\frac{A_{m, n}}{A_{m-k, n-l}}\right)^{\frac{k+l}{2 k l}} \\
& =k l \bar{a}^{-\frac{k^{2}+l^{2}}{2 k l}}\left(\frac{A_{m, n}}{A_{m-k, n-l}}\right)^{\frac{1}{k}} .
\end{aligned}
$$

Thus

$$
\Psi \leq \frac{\bar{a}^{k+l+1}}{C_{k+l}^{l}}\left(1-2 \bar{a}^{-\frac{(k+1)^{2}}{2 k l}}\left(\frac{A_{m, n}}{A_{m-k, n-l}}\right)^{\frac{1}{\lambda}}\right)
$$

i.e.

$$
\frac{A_{m, n}}{A_{m-k, n-l}} \leq \bar{a}^{k+l} 2^{-\lambda}\left(1-\frac{\Psi C_{k+l}^{l}}{\bar{a}^{k+l+1}}\right)^{\lambda} \leq \frac{\bar{a}^{k+l}}{C_{k+l}^{l}} \frac{\Gamma}{\Psi} .
$$

Hence in view of (1.1) we have

$$
A_{m+1, n}+A_{m, n+1}-\bar{a} A_{m, n} \leq-p_{m, n} A_{m-k, n-l} \leq-\frac{\Psi C_{k+l}^{l}}{\Gamma \bar{a}^{k+l}} p_{m, n} A_{m, n}
$$

Thus

$$
p_{m, n} \leq \frac{\bar{a}^{k+l} \Gamma}{C_{k+l}^{l} \Psi}\left(\bar{a}-\frac{2\left(A_{m+1, n} A_{m, n+1}\right)^{\frac{1}{2}}}{A_{m, n}}\right) \quad \text { for }\left\{\begin{array}{l}
\bar{m}-\tau k \leq m \leq \bar{m} \\
\bar{n}-\tau l \leq n \leq \bar{n}
\end{array}\right.
$$

For $\bar{m}-(\tau-1) k \leq m \leq \bar{m}$ and $\bar{n}-(\tau-1) l \leq n \leq \bar{n}$ we have

$$
\begin{aligned}
\Psi & \leq \frac{1}{k l} \sum_{i=m-k}^{m-1} \sum_{j=n-l}^{n-1} p_{i, j} \\
& \leq \frac{\bar{a}^{k+l} \Gamma}{C_{k+l}^{l} \Psi}\left(\bar{a}-\frac{2}{k l} \sum_{i=m-k}^{m-1} \sum_{j=n-l}^{n-1} \frac{\left(A_{i+1, j} A_{i, j+1}\right)^{\frac{1}{2}}}{A_{i, j}}\right) \\
& \leq \frac{\bar{a}^{k+l+1} \Gamma}{C_{k+l}^{l} \Psi}\left(1-2 \bar{a}^{-\frac{(k+l)^{2}}{2 k l}}\left(\frac{A_{m, n}}{A_{m-k, n-l}}\right)^{\frac{1}{\lambda}}\right)
\end{aligned}
$$

Hence

$$
\frac{A_{m, n}}{A_{m-k, n-1}} \leq \frac{\bar{a}^{k+l}}{2^{\lambda}}\left(1-\frac{C_{k+1}^{l} \Psi^{2}}{\bar{a}^{k+l+1} \Gamma}\right)^{\lambda} \leq \frac{\bar{a}^{k+l}}{C_{k+l}^{l}}\left(\frac{\Gamma}{\Psi}\right)^{2} .
$$

By induction, we can get

$$
\frac{A_{m, n}}{A_{m-k, n-l}} \leq \frac{\bar{a}^{k+l}}{C_{k+l}^{l}}\left(\frac{\Gamma}{\Psi}\right)^{r} \quad \text { for } \quad\left\{\begin{array}{l}
\bar{m}-k \leq m \leq \bar{m} \\
\bar{n}-l \leq n \leq \bar{n}
\end{array}\right.
$$

The proof is complete 
In view of Lemma 2.9 and Lemma 3.1, we see that if we let $\bar{a}, \tau, \Psi$ and $\Gamma=\frac{\bar{a}^{k+1+1} \lambda^{\lambda}}{2^{\lambda}(1+\lambda)^{1+\lambda}}$ be positive such that

$$
\left(\frac{k l \Psi}{2 \bar{a}^{k+l+1}}\right)^{4} \geq \frac{1}{C_{k+l}^{l}}\left(\frac{\Gamma}{\Psi}\right)^{\top}
$$

and $\bar{m}, \bar{n}$ be positive integers such that

$$
\begin{aligned}
p_{i, j} \geq 0 \text { and } \quad 0<a_{i, j} \leq \bar{a} & \text { for }\left\{\begin{array}{l}
\bar{m}-(\tau+2) k \leq i \leq \bar{m}+k+l \\
\bar{n}-(\tau+2) l \leq j \leq \bar{n}+k+l
\end{array}\right. \\
\frac{1}{k l} \sum_{i=m-k}^{m-1} \sum_{j=n-l}^{n-1} p_{i, j} \geq \Psi & \text { for }\left\{\begin{array}{l}
\bar{m}-(\tau-1) k \leq m \leq \bar{m}+k \\
\bar{n}-(\tau-1) l \leq n \leq \bar{n}
\end{array}\right.
\end{aligned}
$$

then equation (1.1) has no solution $\left\{A_{i, j}\right\}$, which satisfies

$$
A_{i, j}>0 \quad \text { for }\left\{\begin{array}{l}
\bar{m}-(\tau+3) k \leq i \leq \bar{m}+k+l+1 \\
\bar{n}-(\tau+3) l \leq j \leq \bar{n}+k+l+1
\end{array}\right.
$$

The follwing theorem now follows easily.

Theorem 3.2. Assume that $\lambda=\frac{2 k l}{k+1}, \Gamma=\frac{\frac{\dot{a}}{k}^{k+1+1} \lambda^{\lambda}}{2^{\lambda}(1+\lambda)^{1+\lambda}}, \bar{a}>0, \Psi>0, \tau>0$ and (3.1) holds. Let $q=\left\{q_{m, n}\right\}$ be a double sequence defined by

$$
q_{m, n}=\frac{1}{k l} \sum_{i=m-k}^{m-1} \sum_{j=n-l}^{n-1} p_{i, j} \quad \text { for }\left\{\begin{array}{l}
m=k+1, k+2, \ldots \\
n=l+1, l+2, \ldots
\end{array}\right.
$$

Assume further that

$$
\mu^{*}(p<0)=\bar{B}, \quad \mu^{*}(a>\bar{a} \text { or } a \leq 0)=\bar{A}, \quad \mu_{*}(q<\Psi)=\bar{D}
$$

and

$$
((\tau+3) k+l+1)((\tau+3) l+k+1)(\bar{A}+\bar{B}+\bar{D})<1 .
$$

Then equation (1.1) has no frequently positive solutions.

Proof. Supposing the contrary, let $A=\left\{A_{i, j}\right\}$ be a solution of equation (1.1) such that $\mu^{*}(A \leq 0)=0$. By Lemmas $2.1-2.4$ we get

$$
\begin{aligned}
& \mu_{*}\left(\mathbb{N}^{2} \backslash \sum_{i=-k-l-1}^{(r+3) k} \sum_{j=-k-l-1}^{(r+3) l} X^{i} Y^{j}(A \leq 0)\right) \\
& \quad+\mu^{*}\left(\mathbb{N}^{2} \backslash \sum_{i=-k-l j=-k-l}^{(r+2) k} \sum_{(r+2) l}^{(r+1} X^{i}(a \leq 0 \text { or } a>\bar{a} \text { or } p<0 \text { or } q<\bar{\Psi})\right)
\end{aligned}
$$




$$
\begin{aligned}
= & 2-\mu_{*}\left(\sum_{i=-k-l}^{(\tau+2) k} \sum_{j=-k-l}^{(\tau+2) l} X^{i} Y^{j}(a \leq 0 \text { or } a>\bar{a} \text { or } p<0 \text { or } q<\Psi)\right) \\
& -\mu^{*}\left(\sum_{i=-k-l-1}^{(\tau+3) k} \sum_{j=-k-l-1}^{(\tau+3) l} X^{i} Y^{j}(A \leq 0)\right) \\
\geq & 2-((\tau+3) k+l+1)((\tau+3) l+k+1) \\
& \times \mu_{*}(a \leq 0 \text { or } a>\bar{a} \text { or } p<0 \text { or } q<\Psi) \\
& -((\tau+4) k+l+2)((\tau+4) l+k+2) \mu^{*}(A \leq 0) \\
\geq & 2-((\tau+3) k+l+1)((\tau+3) l+k+1)(\bar{A}+\bar{B}+\bar{D}) \\
> & 1 .
\end{aligned}
$$

Thus

$$
\begin{gathered}
\left(\mathbb{N}^{2} \backslash \sum_{i=-k-l-1}^{(\tau+3) k} \sum_{j=-k-l-1}^{(\tau+3) l} X^{i} Y^{j}(A \leq 0)\right) \\
\bigcap\left(\mathbb{N}^{2} \backslash \sum_{i=-k-l}^{(\tau+2) k} \sum_{j=-k-l}^{(\tau+2) l} X^{i} Y^{j}(a \leq 0 \text { or } a>\bar{a} \text { or } p<0 \text { or } q<\Psi)\right)
\end{gathered}
$$

is an infinite subset of $\mathbb{N}^{2}$. In view of (2.1), we see that there are positive integers $\bar{m}$ and $\bar{n}$ such that

$$
\begin{aligned}
& A_{m, n}>0 \quad \text { for }\left\{\begin{array}{l}
\bar{m}-(\tau+3) k \leq m \leq \bar{m}+k+l+1 \\
\bar{n}-(\tau+3) l \leq n \leq \bar{n}+k+l+1
\end{array}\right. \\
& a_{m, n} \in(0, \bar{a}], p_{m, n} \geq 0, q_{m, n} \geq \Psi \quad \text { for }\left\{\begin{array}{l}
\bar{m}-(\tau+2) k \leq m \leq \bar{m}+k+l \\
\bar{n}-(\tau+2) l \leq n \leq \bar{n}+k+l
\end{array}\right.
\end{aligned}
$$

which is a contradiction. The proof is complete

Corollary 3.1. Assume that

$$
\lambda=\frac{2 k l}{k+l} \quad \text { and } \quad \Gamma=\frac{\bar{a}^{k+l+1} \lambda^{\lambda}}{2^{\lambda}(1+\lambda)^{1+\lambda}} .
$$

and $a_{m, n} \equiv \vec{a}>0$ for $m, n \geq 0$. If $p_{m, n} \geq 0$ and

$$
\liminf _{m, n \rightarrow \infty} \frac{1}{k l} \sum_{i=m-k}^{m-1} \sum_{j=n-l}^{n-1} p_{i, j}>\Gamma,
$$

then every solution of equation (1.1) is frequently oscillatory. and $n$

Proof. It is easy to see that there exist $\tau>0$ and $\Psi>\Gamma$ such that for all large $m$

$$
q_{m, n}=\frac{1}{k l} \sum_{i=m-k}^{m-1} \sum_{j=n-l}^{n-1} p_{i, j} \geq \Psi>\Gamma
$$


and (3.1) holds, i.e.

$$
\left(\frac{k l \Psi}{2 \bar{a}^{\hat{k}+l+1}}\right)^{4} \geq \frac{1}{C_{k+l}^{l}}\left(\frac{\Gamma}{\Psi}\right)^{\top}
$$

Since

$$
\mu^{*}(a>\bar{a} \text { or } a \leq 0)=0, \quad \mu^{*}(p<0)=0, \quad \mu_{*}(q<\Psi)=0
$$

then

$$
((\tau+3) k+l+1)((\tau+3) l+k+1)(0+0+0)=0<1 .
$$

By Theorem 3.2, every solution of equation (1.1) is frequently oscillatory

From Corollary 3.1, the following result is obvious.

Corollary 3.2. If $a_{m, n} \equiv 1$ and $p_{m, n} \geq 0$, and

$$
\liminf _{m, n \rightarrow \infty} \frac{1}{k l} \sum_{i=m-k}^{m-1} \sum_{j=n-l}^{n-1} p_{i, j}>\frac{\lambda^{\lambda}}{2^{\lambda}(1+\lambda)^{1+\lambda}}
$$

where $\lambda=\frac{2 k l}{k+l}$, then equation (1.1) has no frequently positive solutions, and hence it has no eventually positive or eventually negative solutions.

Remark. Corollary 3.2 improves [10: Theorem 3.4].

From Lemma 2.6 we can obtain the following result.

Theorem 3.3. Assume that $\bar{p}$ and $\bar{a}$ are two positive numbers and $\omega$ is a nonnegative number. Let $\mu^{*}(a \leq 0$ or $a>\bar{a})=\bar{A}$ and $\mu_{*}(p<\bar{p})=\bar{B}$. Further assume that

$$
\bar{p}(k+l+1) \sqrt{C_{2 k+2 l}^{2 l}} \geq \bar{a}^{k+l+1}
$$

and

$$
(3 k+2 l+1)(3 l+2 k+1)(\bar{A}+\bar{B})+(4 k+2 l+2)(4 l+2 \dot{k}+2) \omega<1 .
$$

Then every solution of equation (1.1) is frequently oscillatory of upper degree $\omega$.

Proof. Without loss of generality, assume that $\min (k, l)=l$. Suppose to the contrary there is a frequently positive solution $A=\left\{A_{m, n}\right\}$ with upper degree $\omega$ such that $\mu^{*}(A \leq 0) \leq \omega$. In view of Lemmas $2.1-2.3$ and (2.4) we have

$$
\begin{aligned}
\mu^{*}\left(\mathbb{N}^{2} \backslash\right. & \left.\sum_{i=-2 k-l}^{k+l} \sum_{j=-k-2 l}^{k+l} X^{i} Y^{j}(a \leq 0 \text { or } a>\bar{a} \text { or } p<\bar{p})\right) \\
& +\mu_{*}\left(\mathbb{N}^{2} \backslash \sum_{i=-2 k-l-1}^{2 k+l} \sum_{j=-k-2 l-1}^{k+2 l} X^{i} Y^{j}(A \leq 0)\right) \\
& =2-\mu_{*}\left(\sum_{i=-2 k-l}^{k+l} \sum_{j=-k-2 l}^{k+l} X^{i} Y^{j}(a \leq 0 \text { or } a>\bar{a} \text { or } p<\bar{p})\right. \\
& -\mu^{*}\left(\sum_{i=-2 k-l-1}^{2 k+l} \sum_{j=-k-2 l-1}^{2 l+k} X^{i} Y^{j}(A \leq 0)\right)
\end{aligned}
$$




$$
\begin{aligned}
& \geq 2-(3 k+2 l+1)(2 k+3 l+1) \mu_{*}(a \leq 0 \text { or } a>\bar{a} \text { or } p<\bar{p}) \\
& -(4 k+2 l+2)(2 k+4 l+2) \mu^{*}(A \leq 0) \\
\geq & 2-(3 k+2 l+1)(2 k+3 l+1)(\bar{A}+\bar{B})-(4 k+2 l+2)(2 k+4 l+2) \omega \\
> & 1 .
\end{aligned}
$$

Hence by Lemma 2.2 ,

$$
\begin{gathered}
\left(\mathbb{N}^{2} \backslash \sum_{i=-2 k-l}^{k+l} \sum_{j=-k-2 l}^{k+l} X^{i} Y^{j}(a \leq 0 \text { or } a>\bar{a} \text { or } p<\bar{p})\right) \\
\bigcap\left(\mathbb{N}^{2} \backslash \sum_{i=-2 k-l-1}^{2 k+l} \sum_{j=-k-2 l-1}^{2 l+k} X^{i} Y^{j}(A \leq 0)\right)
\end{gathered}
$$

is an infinite subset of $\mathbb{N}^{2}$, which together with (2.1) implies that there is a lattice point $(\bar{m}, \bar{n})$ such that

$$
A_{m, n}>0 \quad \text { for }\left\{\begin{array}{l}
m \in\{\bar{m}-2 k-l, \ldots, \bar{m}+2 k+l+1\} \\
n \in\{\bar{n}-2 l-k, \ldots, \bar{n}+k+2 l+1\}
\end{array}\right.
$$

$0<a_{m, n} \leq \bar{a} \quad$ and $\quad p_{m, n} \geq \bar{p} \quad$ for $\left\{\begin{array}{l}m \in\{\bar{m}-k-l, \ldots, \bar{m}+2 k+l\} \\ n \in\{\bar{n}-k-l, \ldots, \bar{n}+k+2 l\}\end{array}\right.$

By Lemmas 2.4 and 2.6 we obtain

$$
\begin{aligned}
\bar{a}^{k+l+1} A_{\bar{m}, \bar{n}} & >(k+l+1) \bar{p} \sum_{j=0}^{k+l} C_{k+l}^{j} A_{\bar{m}+l-j, \bar{n}+j-l} \\
& \geq(k+l+1) \bar{p} \sum_{j=0}^{2 l} C_{k+l}^{j} A_{\bar{m}+l-j, \bar{n}+j-l} \\
& \geq \frac{\bar{p}^{2}(k+l+1)^{2}}{\bar{a}^{k+l+1}} \sum_{j=0}^{2 l} C_{k+l}^{j} C_{k+l}^{2 l-j} A_{\bar{m}, \bar{n}}
\end{aligned}
$$

By the inequality $\sum_{j=0}^{2 l} C_{k+1}^{j} C_{k+1}^{2 l-j}=C_{2 k+2 l}^{2 l}$ we have

$$
\bar{a}^{k+l+1}>(k+l+1) \bar{p} \sqrt{C_{2 k+2 l}^{2 l}}
$$

which is a contradiction. The proof is complete

Example 3.1. Consider the partial difference equation

$$
A_{m+1, n}+A_{m, n+1}-2 A_{m, n}+p_{m, n} A_{m-1, n-1}=0
$$


where

$$
p_{m, n}= \begin{cases}-1 & \text { if } m=8 k \text { and } n=8 l \quad\left(k, l \in \mathbb{N}_{0}\right) \\ \frac{4}{3} & \text { otherwise. }\end{cases}
$$

Take $0 \leq \omega<\frac{1}{48}$. Since

$$
\mu^{*}(a>2 \text { or } a \leq 0)=0, \quad \mu^{*}\left(p<\frac{4}{3}\right)=\frac{1}{64}, \quad 16 \times \frac{1}{64}+36 \omega<1, \quad \frac{4}{3} \times \frac{3 !}{2^{3}}=1,
$$

then by Theorem 3.1 every solution of equation (3.2) is frequently oscillatory of lower degree $\omega$, and hence oscillatory.

Example 3.2. Consider the difference equation

$$
A_{m+1, n}+A_{m, n+1}-A_{m, n}+P_{m, n} A_{m-1, n-2}=0
$$

where

$$
P_{m, n}= \begin{cases}0 & \text { if } m=9 k \text { and } n=9 l \quad\left(k, l \in \mathbb{N}_{0}\right) \\ \frac{1}{12} & \text { otherwise. }\end{cases}
$$

Then

$$
\mu^{*}(a \leq 0 \text { or } a>1)=0 \quad \text { and } \quad \mu_{*}\left(p<\frac{1}{12}\right)=\frac{1}{81} .
$$

Taking $0 \leq \omega<\frac{1}{1080}$, then $72 \times \frac{1}{81}+120 \omega<1$ and $\frac{1}{12} \times 4 \times \sqrt{C_{6}^{2}}>1$. Therefore by Theorem 3.3 every solution of equation (3.3) is frequently oscillatory of upper degree $\omega$ and hence oscillatory. But Theorem 3.1 is not available here.

\section{References}

[1] Agarwal, R. P.: Difference Equations and Inequalities. New York: Marcel Dekker 1992.

(2) Cheng, S. S. and B. G.Zhang: Qualitative theory of partial difference equations. Part I: Oscillation of nonlinear partial difference equations. Tamkang J. Math. 25 (1994), 279 288.

[3] Courant, R., Friedrichs, K. and H. Lewy: On partial difference equations of mathematical physics. IBM J. 11 (1967), $215-234$.

[4] Kelley, W. G. and A. C. Peterson: Difference Equations. New York: Acad. Press 1991.

[5] Li, X.-P.: Partial difference equations used in the study of molecular orbits (in Chinese). Acta Chimica SINICA 40 (1982), 688 - 698.

[6] Tian, C. J., Xie, S. L. and S. S. Cheng: Measures for oscillatory sequences. Comp. Math. Appl. (to appear).

[7] Tramov, M. I.: Oscillation of partial differential equations with deviating arguments (in Russian). Diff. Uravn. 20 (1984), 721 - 723.

[8] Zhang, B. G. and S. T. Liu: Oscillation of partial difference equations. PanAmer. Math. J. $5(1995) 2,61-70$.

[9] Zhang, B. G. and S. T. Liu: On the oscillation of two partial difference equations. J. Math. Anal. Appl. 206 (1997), 480 - 492.

[10] Zhang, B. G., Liu, S. T. and S. S. Cheng: Oscillation of a class of delay partial difference equations. J. Difference Equ. Appl. 1 (1995), 215 - 226. 
[11] Zhang, B. G. and J. S. Yu: Linearized oscillation theorems for certain nonlinear delay partial difference equations. Comp. Math. Applic. 35 (1998)4, $111-116$.

Received 29.04.1998: in revised form 16.12.1998 